Research Article

\title{
Dynamic Evaluation Method of the EW-AHP Attribute Identification Model for the Tunnel Gushing Water Disaster under Interval Conditions and Applications
}

\author{
Bo Wu $\mathbb{D}^{1,2,3}$ Huihao Chen $\mathbb{D}^{1},{ }^{1}$ Wei Huang $\mathbb{D}^{1},{ }^{1}$ and Guowang Meng $\mathbb{D}^{1}$ \\ ${ }^{1}$ College of Civil Engineering and Architecture, Guangxi University, Nanning 530004, Guangxi, China \\ ${ }^{2}$ School of Civil and Architectural Engineering, East China University of Technology, Nanchang 330013, Jiangxi, China \\ ${ }^{3}$ School of Architectural Engineering, Guangzhou City Construction College, Guangzhou 510925, Guangdong, China
}

Correspondence should be addressed to Huihao Chen; 1910302006@st.gxu.edu.cn and Wei Huang; wei.huang@st.gxu.edu.cn

Received 11 December 2020; Revised 31 March 2021; Accepted 2 April 2021; Published 19 April 2021

Academic Editor: Adrian Neagu

Copyright $(0) 2021$ Bo Wu et al. This is an open access article distributed under the Creative Commons Attribution License, which permits unrestricted use, distribution, and reproduction in any medium, provided the original work is properly cited.

The gushing water disaster in tunnels is a kind of harmful and risky engineering disaster. It has become a key problem to evaluate the risk of tunnel gushing water accurately and objectively. A case study of a typical highway tunnel is performed for theory and practice analysis. For this reason, the risk identification is carried out on the assessed objects, and 10 evaluation indexes are determined. In turn, the risk evaluation index system and classification standard are established. Furthermore, the entropy weight method and the analytic hierarchy process are combined to assign the weight to each evaluation index. Therefore, a dynamic risk assessment system, including the pre-evaluation model and the postevaluation model, is constructed with the attribute identification model. As a result, the tunnel section with a high risk of water inrush is accurately assessed, which is consistent with the construction situation on site. Moreover, it is verified that the assessment results are reliable, which can provide a reference for the similar projects.

\section{Introduction}

China is the fastest developing country in tunneling construction [1]. However, the safety of tunneling construction is particularly prominent and has attracted much attention. In particular, the tunnel water inrush is a kind of engineering disaster which is difficult to predict. Therefore, the safety risk assessment of tunneling construction is widely practiced. By combining the interval analytic hierarchy process (I-AHP) with the technique for order preference by similarity to an ideal solution (TOPSIS), Lin et al. [2] proposed a novel fuzzy model which identified high-risk factors during excavations in urban karst geological environments. In addition, the theory of risk assessment has also developed rapidly. For example, Lin et al. [3] conducted research on the excavation system based on fuzzy set theory and machine learning methods. Furthermore, the development of assessment theory also promotes the application of safety assessment of the tunneling water inrush. For instance, Li et al. [4] established a mechanical model of the minimum safe thickness of the rock burst prevention disk and carried out a series of model tests on the rock burst prevention disk in front of the tunnel face to find out the influence rules of various factors on the tunnel gushing water disaster. Additionally, Zhu et al. [5] focused on the study of the influence of the geological influencing factors on the tunnel gushing water risk and, based on this, established the risk assessment model of tunnel gushing water based on the weighted average method, etc.

At present, the lack of research on the systematic evaluation of the tunnel gushing water risk assessment theory has limited its popularization. Furthermore, many theories are static evaluation methods, which ignore that the evaluation index values are not always consistent with the previous ones during tunnel excavation. Therefore, a brand new risk assessment model is established, where the 
subjective and objective weighting complex method are used to calculate the indexes' weight. Thus, the evaluation indexes can be assigned as interval or numerical values, which can change dynamically in real time according to the construction situation.

A case study of a typical highway tunnel in Fujian province is performed for theory and practice analysis. Through the analysis of its engineering characteristics and special geological conditions, a dynamic evaluation model of the highway tunnel water inrush risk is proposed based on the entropy weight method.

\section{Methods}

2.1. Improved Theoretical Model of Attribute Interval Identification. The problem of the system comprehensive evaluation is the measurement of the qualitative description. Attribute mathematics is a new branch of mathematics which appears to solve many systematic evaluation problems. The theoretical model of the attribute interval identification is developed on the basis of attribute mathematics. Suppose that evaluation objects' space $X=\left\{x_{1}, \ldots\right.$, $\left.x_{i}, \ldots, x_{n}\right\}$, where all the evaluation objects could be $x_{i}(i=1,2, \ldots, n) . I_{i j}$ is the $j^{\text {th }}$ evaluation index of the evaluation object $x_{i} . F$ is the attribute interval space of $X$. $\left(R_{1}, R_{2}, \ldots, R_{k}, \ldots, R_{K}\right)$ is an ordered partition class of $F$, where $R_{1}<R_{2}<\cdots<R_{k}[6]$.

2.1.1. Analysis of the Single-Index Attribute Measurement. It can be supposed that the value of the evaluation object $x_{i}$ of the evaluation index $I_{i j}$ is $t_{i j}$, and the attribute measure function represents the situation that the attribute measure $\mu_{i j}$ changes with the value $t_{i j}$ of the evaluation index $I_{i j}$. The grading standards of the evaluation index are shown in Table 1 . The main steps of the data processing are as follows:

$$
\begin{aligned}
b_{j k} & =\frac{a_{j(k-1)}+a_{j k}}{2}, \\
d_{j k} & =\min \left\{\left|b_{j k}-a_{j k}\right|,\left|b_{j(k+1)}-a_{j k}\right|\right\},
\end{aligned}
$$

where $k=1,2, \ldots, K$ and $j=1,2, \ldots, m$ in equation (1) and $k=1,2, \ldots,(K-1)$ and $j=1,2, \ldots, m$ in equation (2).

From [7-9], when $a_{j 0}<a_{j 1}<\cdots a_{j k} \cdots a_{j K}$, the singleindex attribute measure function $\mu_{i j k}(t)$ can be expressed as follows:

$$
\begin{aligned}
& \mu_{i j 1}(t)= \begin{cases}1, & t_{j}<a_{j 1}-d_{j 1}, \\
\frac{a_{j 1}+d_{j 1}-t_{j}}{2 d_{j 1}}, & a_{j 1}-d_{j 1} \leq t_{j} \leq a_{j 1}+d_{j 1}, \\
0, & t_{j}>a_{j 1}+d_{j 1},\end{cases} \\
& \mu_{i j}(t)= \begin{cases}0, & t_{j}<a_{j(k 1)}-d_{j(k-1)}, \\
\frac{t_{j}<a_{j(k 1)}-d_{j(k-1)}}{2 d_{j(k-1)}}, & a_{j(k-1)}-d_{j(k-1)} \leq t_{j} \leq a_{j(k-1)}+d_{j(k-1)}, \\
1, & a_{j(k-1)}+d_{j(k-1)}<t_{j}<a_{j k}-d_{j k}, \\
\frac{a_{j k}+d_{j k}-t_{j}}{2 d_{j k},} & a_{j k}-d_{j k} \leq t_{j} \leq a_{j k}+d_{j k}, \\
0, & t_{j}<a_{j(K-1)}-d_{j(K-1)},\end{cases} \\
& \mu_{i j K}(t)= \begin{cases}0, & t_{j}<a_{j(K-1)}-d_{j(K-1)}, \\
\frac{t_{j}-a_{j(K-1)}+d_{j(K-1)}}{2 d_{j(K-1)}}, & a_{j(K-1)}-d_{j(K-1)} \leq t_{j} \leq a_{j(K-1)}+d_{j(K-1)}, \\
1, & t_{j}>a_{j(K-1)}+d_{j(K-1)} .\end{cases}
\end{aligned}
$$


TABLE 1: Grading standards for the evaluation index.

\begin{tabular}{|c|c|c|c|c|c|}
\hline \multirow{2}{*}{ Evaluation index $I_{i}$} & \multirow{2}{*}{ Evaluation index value $t_{i}$} & \multicolumn{4}{|c|}{ Order of evaluation } \\
\hline & & $R_{1}$ & $R_{2}$ & $\ldots$ & $R_{k}$ \\
\hline$\overline{I_{i 1}}$ & $t_{i 1}$ & $a 10 \sim a 11$ & $a 11_{\sim} a 12$ & $\ldots$ & $a 1(k-1) \sim a 1 k$ \\
\hline $\begin{array}{c}I_{i 2} \\
:\end{array}$ & $t_{i 2}$ & $a 20 \sim a 21$ & $a 21 \sim a 22$ & $\cdots$ & $a 2(k-1) \sim a 2 k$ \\
\hline $\begin{array}{c}\vdots \\
I_{i j}\end{array}$ & $\begin{array}{l}: \\
t_{i j}\end{array}$ & $\begin{array}{c}: \\
a j 0 \sim a j 1\end{array}$ & $\begin{array}{c}: \\
a j 1\end{array}$ & $\ddots$ & $a j(k-1) \sim a j k$ \\
\hline $\begin{array}{c}\vdots \\
I_{i m}\end{array}$ & $\begin{array}{c}\vdots \\
t_{i m}\end{array}$ & $\begin{array}{c}\vdots \\
a m 0 \sim a m 1\end{array}$ & $\begin{array}{c}\vdots \\
\operatorname{am1} \sim \operatorname{am} 2\end{array}$ & $\ddots$ & $\begin{array}{c}\vdots \\
\operatorname{am}(k-1) \sim a m k\end{array}$ \\
\hline
\end{tabular}

From $[9,10]$, when $a_{j 0}<a_{j 1}<\cdots a_{j k} \cdots<a_{j K}$, the single-index attribute measure function $\mu_{i j k}(t)$ can be expressed as follows:

$$
\begin{aligned}
& \mu_{i j 1}(t)= \begin{cases}1, & t_{j}<a_{j 1}-d_{j 1}, \\
\frac{a_{j 1}+d_{j 1}+d_{j 1}}{2 d_{j 1}}, & a_{j 1}-d_{j 1} \leq t_{j} \leq a_{j 1}+d_{j 1}, \\
0, & t_{j}>a_{j 1}+d_{j 1},\end{cases} \\
& \mu_{i j}(t)= \begin{cases}0, & t_{j}<a_{j k}-d_{j k}, \\
\frac{t_{j}<a_{j(k-1)}-d_{j(k-1)}}{2 d_{j(k-1)},} & a_{j k}-d_{j k} \leq t_{j} \leq a_{j k}+d_{j k}, \\
1, & a_{j k}+d_{j k}<t_{j}<a_{j(k-1)}-d_{j(k-1)}, \\
\frac{a_{j k}+d_{j k}-t_{j}}{2 d_{j}}, & a_{j(k-1)}-d_{j(k-1)} \leq t_{j} \leq a_{j(k-1)}+d_{j(k-1)}, \\
0, & t_{j}>a_{j(k-1)}-d_{j(k-1)},\end{cases} \\
& \mu_{i j K}(t)= \begin{cases}1, & t_{j}<a_{j(K-1)}-d_{j(K-1)}, \\
\frac{a_{j(K-1)}+d_{j(K-1)}-t_{j}}{2 d_{j(K-1)},} & a_{j(K-1)}-d_{j(K-1)} \leq t_{j} \leq a_{j(K-1)}+d_{j(K-1)}, \\
0, & t_{j}>a_{j(K-1)}+d_{j(K-1)},\end{cases}
\end{aligned}
$$

where $i=1,2, \ldots, n ; j=1,2, \ldots, m$; and $k=1,2, \ldots, K$.

2.1.2. Multi-Index Comprehensive Attribute Measurement Analysis. (1) The evaluation index value is an interval-type index: from [9], let $S_{i j k}(t)$ be the interval metric function of the single-index attribute, and let $t_{j}^{+}$and $t_{j}^{-}$be the upper and lower limits of the evaluation index, respectively. When $\alpha_{j 0}>\alpha_{j 1}>\cdots \alpha_{j k} \cdots>\alpha_{j K}$, the interval metric function $S_{i j k}(t)$ of the single-index attribute can be expressed as follows: 


$$
\begin{aligned}
& s_{i j 1}(t)= \begin{cases}0, & t_{j}^{-} \geq a_{j 1}+d_{j 1}, \\
\int_{t_{j}^{-}}^{a_{j 1}+d_{j 1}} \mu_{i j 1}(t) \mathrm{d} t, & t_{j}^{-} \leq a_{j 1}+d_{j 1} \leq t_{j}^{+}, \\
\int_{t_{j}^{-}}^{t_{j}^{+}} \mu_{i j 1}(t) \mathrm{d} t, & t_{j}^{+} \leq a_{j 1}+d_{j 1},\end{cases} \\
& S_{i j k}(t)= \begin{cases}0, & t_{j}^{+} \leq a_{j(k-1)}-d_{j(k-1)}, \\
\int_{a_{j(k-1)}-d_{j(k-1)}}^{t_{j}^{+}} \mu_{i j k}(t) \mathrm{d} t, & t_{j}^{-} \leq a_{j(k-1)}-d_{j(k-1)} \leq t_{j}^{+} \leq a_{j k}+d_{j k}, \\
t_{j}^{+} \mu_{i j k}(t) \mathrm{d} t, & a_{j(k-1)}-d_{j(k-1)} \leq t_{j}^{-} \leq t_{j}^{+} \leq a_{j k}+d_{j k}, \\
\int_{t_{j}^{-}}^{a_{j k}+d_{j k}} \mu_{i j k}(t) \mathrm{d} t, & a_{j(k-1)}-d_{j(k-1)} \leq t_{j}^{-} \leq a_{j k}+d_{j k} \leq t_{j}^{+}, \\
0, & t_{j}^{-} \geq a_{j k}+d_{j k},\end{cases} \\
& s_{i j K}(t)= \begin{cases}0, & t_{j}^{+} \leq a_{j(K-1)}-d_{j(K-1)}, \\
\int_{a_{j(k-1)}-d_{j(k-1)}}^{t_{j}^{+}} \mu_{i j K}(t) \mathrm{d} t, & t_{j}^{-} \leq a_{j(K-1)}-d_{j(K-1)} \leq t_{j}^{+}, \\
\int_{t_{j}^{-}}^{t_{j}^{+}} \mu_{i j K}(t) \mathrm{d} t, & t_{j}^{-} \geq a_{j(K-1)}-d_{j(K-1)} .\end{cases}
\end{aligned}
$$

When $\alpha_{j 0}>\alpha_{j 1}>\cdots \alpha_{j k} \cdots>\alpha_{j K}$, the interval metric function $S_{i j k}(t)$ of the single-index attribute can be expressed as follows:

$$
\begin{aligned}
& s_{i j 1}(t)= \begin{cases}\int_{t_{j}^{-}}^{t_{j}^{+}} \mu_{i j 1}(t) \mathrm{d} t, & t_{j}^{-} \geq a_{j 1}-d_{j 1}, \\
\int_{a_{j 1}-d_{j 1}}^{t_{j}^{+}} \mu_{i j 1}(t) \mathrm{d} t, & t_{j}^{-} \leq a_{j 1}-d_{j 1} \leq t_{j}^{+}, \\
0, & t_{j}^{+} \leq a_{j 1}+d_{j 1},\end{cases} \\
& S_{i j k}(t)= \begin{cases}0, & t_{j}^{+} \leq a_{j k}-d_{j k}, \\
\int_{a_{j k}-d_{j k}}^{t_{j}^{+}} \mu_{i j k}(t) \mathrm{d} t, & t_{j}^{-} \leq a_{j k}-d_{j k} \leq t_{j}^{+} \leq a_{j k}+d_{j k}, \\
\int_{t_{j}^{-}}^{t_{j}^{+}} \mu_{i j k}(t) \mathrm{d} t, & a_{j k}-d_{j k} \leq t_{j}^{-} \leq t_{j}^{+} \leq a_{j(k-1)}+d_{j(k-1)}, \\
\int_{t_{j}^{-}}^{a_{j(k-1)}+d_{j(k-1)}} \mu_{i j k}(t) \mathrm{d} t, & a_{j k}-d_{j k} \leq t_{j}^{-} \leq a_{j(k-1)}+d_{j(k-1)} \leq t_{j}^{+}, \\
0, & t_{j}^{-} \geq a_{j(k-1)}+d_{j(k-1)},\end{cases}
\end{aligned}
$$




$$
s_{i j K}(t)= \begin{cases}\int_{t_{j}^{-}}^{t_{j}^{+}} \mu_{i j K}(t) \mathrm{d} t, & t_{j}^{+} \leq a_{j(K-1)}-d_{j(K-1)}, \\ \int_{t_{j}^{-}}^{a_{j(k-1)}-d_{j(k-1)}} \mu_{i j K}(t) \mathrm{d} t, & t_{j}^{-} \leq a_{j(K-1)}+d_{j(K-1)} \leq t_{j}^{+}, \\ 0, & t_{j}^{-} \geq a_{j(K-1)}+d_{j(K-1)} .\end{cases}
$$

The weight of each index can be $\omega=\left\{\omega_{1}, \omega_{2}, \ldots, \omega_{m}\right\}$, $0 \leq \omega_{j} \leq 1, \sum_{i=1}^{m} \omega_{j}=1$. The attribute measure value $\mu_{i k}$ of the evaluation index $I_{i j}$ belonging to the evaluation level $R_{k}$ can be solved by the single-index attribute interval metric function $S_{i j k}(t)$, which can be expressed as follows:

$$
\begin{aligned}
\mu_{i k} & =\sum_{j=1}^{m} \omega_{j} S_{i j k}^{\prime}, \\
S_{i j k}^{\prime} & =\frac{S_{i j k}}{\sum_{k=1}^{K} S_{i j k}} .
\end{aligned}
$$

(2) The evaluation index value is a noninterval-type index: as the weight of each index is $\omega=\left\{\omega_{1}, \omega_{2}, \ldots, \omega_{m}\right\}$, $0 \leq \omega_{j} \leq 1, \sum_{i=1}^{m} \omega_{j}=1$, the attribute measure value can be expressed as follows:

$$
\mu_{i k}=\sum_{j=}^{m} \omega_{j} \mu_{i j k}
$$

2.1.3. Attribute Identification Analysis. The attribute space $F$ of a class on $X$ is set, $R_{1}, R_{2}, \ldots, R_{k}$ is the attribute interval set of $X$, and $F=\cup_{i=1}^{k} R_{i}, R_{i} \cap R_{j}=\varnothing(i \neq j)$ [6]. $\lambda$ is the confidence level, and $0.5<\lambda \leq 1$.

When $R_{1}<R_{2}<\cdots<R_{k}<\cdots R_{k}$

$$
k=\min \left\{l: \sum_{k=l}^{l} \mu_{i k} \geq \lambda, \quad 1 \leq l \leq K\right\} .
$$

When $R_{1}<R_{2}<\cdots<R_{k}<\cdots R_{k}$,

$$
k=\max \left\{l: \sum_{k=l}^{l} \mu_{i k} \geq \lambda, \quad 1 \leq l \leq K\right\},
$$

where $x_{i}$ belongs to risk level $R_{k}$.

2.2. Combination Weighting. Different evaluation indexes have different influence proportions on the risk of the highway tunnel water inrush, so the scientific index weighting method should be adopted. The common methods include the subjective weighting method and the objective weighting method [11]. In order to avoid the result deviation caused by the single weighting method, the combined weighting method is adopted, which takes the subjective and objective factors into consideration. Weight. The entropy weight method [12-14] is an objective method, which can determine the weight of the evaluation index objectively. It is according to the difference degree of the evaluation index value, and it can avoid the deviation caused by the human factors. So, it has higher accuracy. However, the disadvantage is that the importance of the evaluation index itself is ignored, and its function is limited if the difference of the evaluation index value is very small. In the risk assessment of the tunnel gushing water disaster, the sensitivity of the entropy weight method to the difference of the evaluation index value can be used. Thus, the difference degree of the same evaluation index value in different tunnel sections can be used as the danger signal of the tunnel gushing water disaster to some extent. Its main steps are shown as follows:

Step 1: the normalized matrix is constructed, and the normalized matrix is standardized. The normalized matrix $Z=\left(z_{i j}\right)_{n \times m}$ can be obtained. Then, the proportion of the $i^{\text {th }}$ sample value in the $j^{\text {th }}$ index of the term is calculated as follows:

$$
p_{i j}=\frac{z_{i j}}{\sum_{i=1}^{n} z_{i j}} .
$$

Step 2: the information entropy of the evaluation index is calculated as follows:

$$
e_{j}=\frac{\sum_{i=1}^{m} p_{i j} \operatorname{In}\left(p i_{j}\right)}{\operatorname{In} n} .
$$

Step 3: the evaluation index weight is assigned as follows:

$$
\alpha_{j}=\frac{u_{j}}{\sum_{j=1}^{n} u_{j}} .
$$

$u_{j}$ is the difference coefficient of the calculated index, and $u_{j}=1-e_{j}$. The weight vector of the index is $\alpha=\left(\alpha_{1}, \alpha_{2}, \ldots, \alpha_{n}\right)$, and $\sum_{j=1}^{n} \alpha_{j}=1$.

\subsubsection{AHP Method to Determine the Subjective Weight.} Analytic hierarchy process (AHP) [15-17] is a systematic analysis method, which can combine qualitative and quantitative methods organically to simplify complex system evaluation problems. It focuses on qualitative analysis and judgment. However, its shortcoming is that it is too subjective. As for too many indicators, the data statistics are large, and the weight is difficult to determine. The basic idea of the AHP is to compare the importance of multiple factors in pairs to judge each other, which improves the rationality of decision-making to some extent [18]. The main steps are as follows: 
Step 1: the hierarchical relationship is determined among the influencing factors, and the system hierarchy is established.

Step 2: combining with the importance level (Table 2), the evaluation indexes are compared in pairs, and the judgment matrix is constructed. The judgment matrix can be obtained as follows:

$$
R=\left[\begin{array}{cccc}
r_{11} & r_{12} & \cdots & r_{1 K} \\
r_{21} & r_{22} & \cdots & r_{2 K} \\
\vdots & \vdots & \ddots & \vdots \\
r_{m 1} & r_{m 2} & \cdots & r_{m K}
\end{array}\right] .
$$

Step 3: the maximum eigenvalue $\lambda_{\max }$ of the judgment matrix $R$ is calculated, the consistency index CI and consistency ratio $\mathrm{CR}$ are calculated, and the consistency test is conducted.

Step 4: the weight $\beta_{j}$ of each evaluation index is calculated.

2.2.3. Combination Weighting of the Evaluation Index. From [19], the combined weights are calculated as follows:

$$
\omega_{j}=\frac{\alpha_{j} \beta_{j}}{\sum_{i=1}^{m} \alpha_{j} \beta_{j}} .
$$

\section{The Dynamic Risk Assessment Model}

3.1. The Index System and the Grading Standards. The risk factors of the gushing water disaster during highway tunneling present characteristics of fuzziness and uncertainty, which make the risk assessment become a complicated systematic evaluation problem. The adverse geological condition refers to the water-rich condition of the geological structure, which is an important influencing factor for the occurrence of tunnel water inrush. Thus, the water level refers to the elevation difference between the underground water level and tunnel floor, which represents the danger degree of water inrush in a way. Also, the length of the waterbearing layer reflects the spatial distribution of water bearing in the tunnel. Besides, the permeability coefficient of rock strata directly affects the groundwater activity. Moreover, the water inflow in the normal period reflects the water fluctuation of groundwater. Also, the negative topographic area ratio directly affects and reflects the development and occurrence characteristics of underground undesirable geology and groundwater. Except them, the dip angle of the rock stratum is an important factor affecting groundwater flow. Furthermore, the development degree of the layer and interlayer fissure affects the activity degree of groundwater, and it has an important influence on the tunnel water inrush. The situation of water dripping on the wall directly represents the current situation of water inrush after the formation of the tunnel initial support. To some extent, the buried depth of the tunnel represents the danger degree of tunnel water inrush.
As for the case of the highway tunneling project in Fujian province, the evaluation indexes are divided into four levels by combining with the geological prospecting, the geological survey data, and the analysis of statistical data [7, 20-23]. Furthermore, the gushing water disaster risk evaluation index system and the gushing water risk evaluation index grading standard have been established. Some are based on the expert scoring to determine the evaluation index classification, which include the influencing factors of the adverse geological conditions $I_{1}$, the level and interlayer fissure $I_{8}$, and the situation of drop water on the wall $I_{9}$. The index system of the highway tunnel water inrush risk assessment is shown in Figure 1. The grading standards of the highway tunnel water inrush risk assessment index are shown in Table 3.

3.2. Attribute Measure Function. From equations (1)-(8), the single-index attribute measure can be calculated, and the calculation results are summarized as shown in Table 4.

3.3. Attribute Identification Analysis. Referring to the "Highway Bridge and Tunnel Engineering Construction Safety Risk Assessment System and Guide Analysis" [24], the highway tunnel gushing water risk is divided into four levels, so the evaluation set is $R=\left\{\begin{array}{llll}R_{1} & R_{2} & R_{3} & R_{4}\end{array}\right\}$. The specific levels are described as shown in Table 5 .

As the confidence value $\lambda$ in attribute identification is generally $0.6 \sim 0.7, \lambda=0.65$ is adopted in the calculation [10]. It can be seen from equation (18) that when $R_{1}<R_{2}<\cdots<R_{k}<\cdots<R_{k}$,

$$
k=\min \left\{l: \sum_{k=l}^{l} \mu_{i k} \geq 0.65, \quad 1 \leq l \leq 4\right\} \text {, }
$$

where $x_{i}$ belongs to risk level $R_{k}$.

3.4. Dynamic Risk Assessment. The dynamic risk assessment system based on the attribute interval identification model is composed of two parts. One is the preassessment model, and the other one is the postassessment model. The research object of the pre-evaluation model is the unexcavated tunnel section. The evaluation index and the value of the evaluation index are determined. The weight of each evaluation index combination is calculated, and the corresponding interval risk assessment is carried out by the pre-evaluation model. The assessment results can provide guidance for construction safety. The research object of the postevaluation model is the tunnel section under construction. On the basis of the pre-evaluation model, it mainly monitors the sections with high risk of water inrush. The evaluation index value can be adjusted and corrected according to the construction and excavation situation on site. The specific flowchart is shown in Figure 2.

The specific steps are as follows:

Step 1: segment the similar terrain and the geological conditions of the unexcavated tunnel section.

Step 2: determine the evaluation index and its value. 
TABLE 2: Importance level table.

\begin{tabular}{lc}
\hline Value & Difference in importance \\
\hline 1 & Indicator $i$ is as important as indicator $j$ \\
3 & Index $i$ is slightly more important than index $j$ \\
5 & Indicator $i$ is significantly more important than indicator $j$ \\
9 & Indicator $i$ is strongly more important than indicator $j$ \\
$2,4,6,8$ & Indicator $i$ is extremely important than indicator $j$ \\
Explanation & Intermediate value between the above adjacent judgments \\
\hline
\end{tabular}

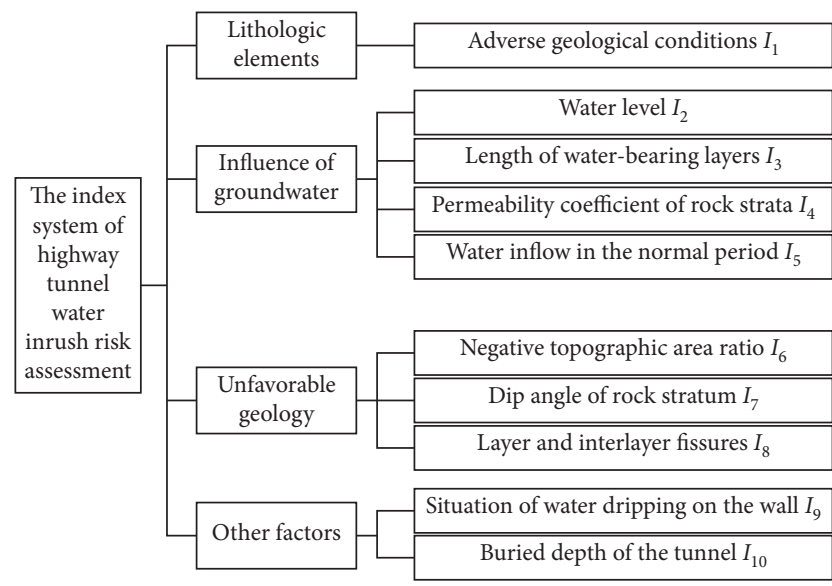

Figure 1: The index system.

TABLE 3: The index grading standards.

\begin{tabular}{lcccc}
\hline Evaluation index & $R_{1}$ & $R_{2}$ & $R_{3}$ & $R_{4}$ \\
\hline$I_{1}$ & $35 \sim 60$ & $60 \sim 70$ & $70 \sim 85$ & $85 \sim 100$ \\
$I_{2}(\mathrm{~m})$ & $h<10$ & $10 \leq h<30$ & $30 \leq h<60$ & $h>60$ \\
$I_{3}(\mathrm{~m})$ & $<50$ & $50 \sim 100$ & $100 \sim 200$ & $200 \sim 300$ \\
$I_{4}\left(10^{-5} \mathrm{~cm} / \mathrm{s}\right)$ & $<1$ & $1 \sim 1000$ & $1000 \sim 100000$ & $>100000$ \\
$I_{5}\left(\mathrm{~m}^{3} / \mathrm{d}\right)$ & $<100$ & $100 \sim 500$ & $500 \sim 1000$ & $>1000$ \\
$I_{6}(\%)$ & $<20$ & $20 \sim 40$ & $40 \sim 60$ & $>60$ \\
$I_{7}\left({ }^{\circ}\right)$ & $0 \sim 10$ & $10 \sim 25$ & $25 \sim 45$ & $45 \sim 65$ \\
$I_{8}$ & $35 \sim 60$ & $60 \sim 70$ & $70 \sim 85$ & $85 \sim 100$ \\
$I_{9}$ & $35 \sim 60$ & $60 \sim 70$ & $70 \sim 85$ & $85 \sim 100$ \\
$I_{10}(\mathrm{~m})$ & $<50$ & $50 \sim 100$ & $100 \sim 200$ & $>200$ \\
\hline
\end{tabular}

Step 3: the combined weighting method was used to assign weights to each evaluation index.

Step 4: pre-evaluate the tunnel interval. Combined with equations (1)-(8), the single-index attribute measure analysis is carried out for each evaluation index, and the single-index attribute measure function is calculated. Determine whether the evaluation index is an intervaltype index. If it is an interval-type index, the multiindex comprehensive attribute measurement analysis is conducted according to equations (9)-(16). Otherwise, the multi-index comprehensive attribute measurement analysis is conducted according to equation (17). Finally, the attribute identification analysis combined with the confidence degree is conducted to determine the hazard level of the tunnel water inrush.
Step 5: dynamically revise the evaluation index value according to the construction and excavation situation, and repeat Step 4 to redetermine the hazard level of the tunnel water inrush after the evaluation of the tunnel section.

Step 6: construct and excavate, and at the same time, take preventive measures for risk control, and perform a good record of emergency.

\section{Engineering Applications}

4.1. Engineering Background. A highway tunneling case of the Puyan highway is a double-line separated tunnel in Sanming, Fujian Province. It is designed as a two-way sixlane tunnel. The main structure of the tunnel area is intrusive contact structure. The surrounding rock of the tunnel is mainly medium-weathered rock. Under the influence of the intrusive contact structure, the joints and fissures in rock mass at the tunnel site are developed, and the integrity of the rock mass is poor. The vault is prone to drop blocks, and accidents such as collapse, water gushing, and mud gushing are easy to occur in the construction. Similarly, with the influence of the stratum, lithological and geological structure, topography, and geomorphology, water in the tunnel area is mainly bedrock fissure water. Bedrock fissure water mainly occurs in strongly and moderately weathered bedrock, among which strongly weathered bedrock mass has developed joint fissure and relatively good water enrichment. According to the analysis of exploration and disclosure of the water level, groundwater in most sections of the tunnel is well developed, and the groundwater level in the tunnel area is higher than the designed elevation. The lining of the tunnel adopts the separated structure, in which the open-cut section adopts the integral lining, and the underground section adopts the composite lining. The initial support mainly consists of steel arch, steel mesh, radial anchor, and sprayed concrete. After the formation of the initial support, the water outlet of the tunnel vault at the construction site is relatively obvious, as shown in Figure 3. Therefore, there is a high risk of the water inrush in this section of the tunnel, and the preventive measures should be strengthened during construction. According to the detailed geological survey reports and the design documents, the right line of the tunnel is divided into 5 sections based on the level of the surrounding rock, as shown in Table 6. 


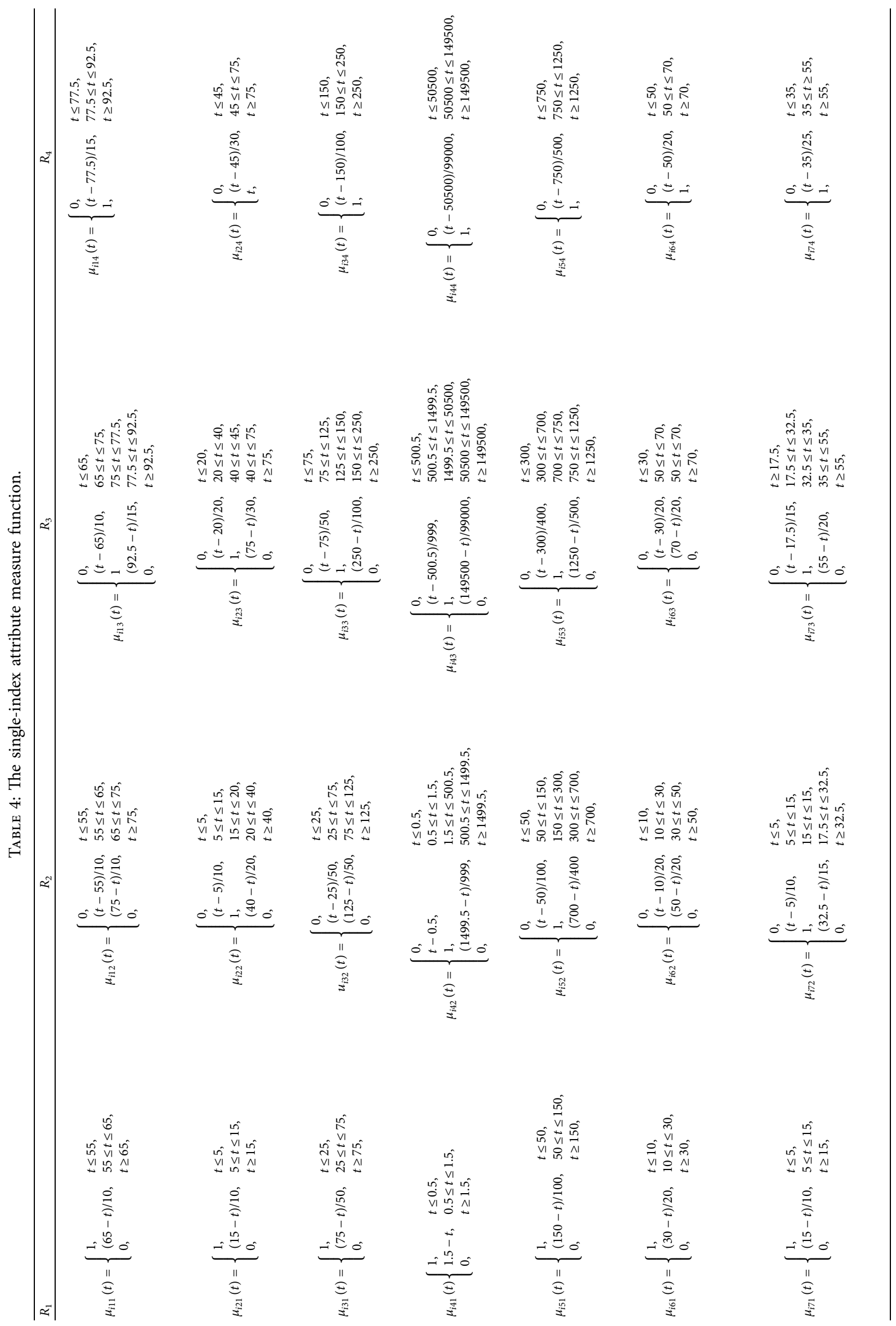




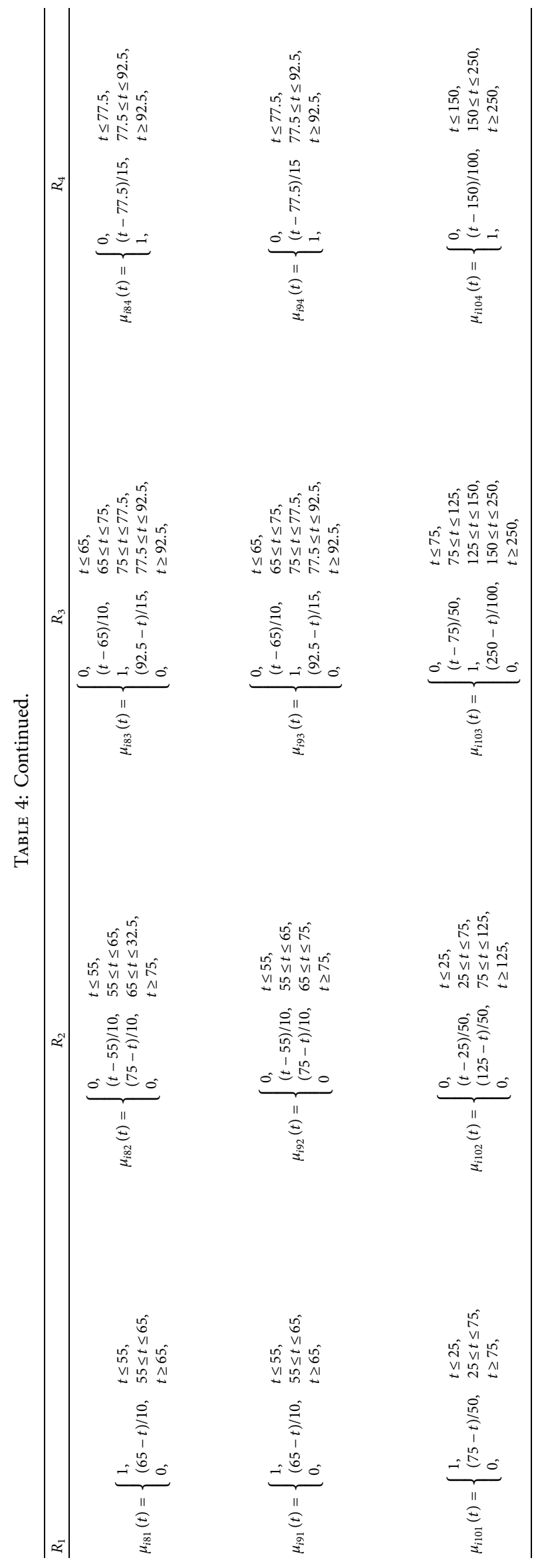




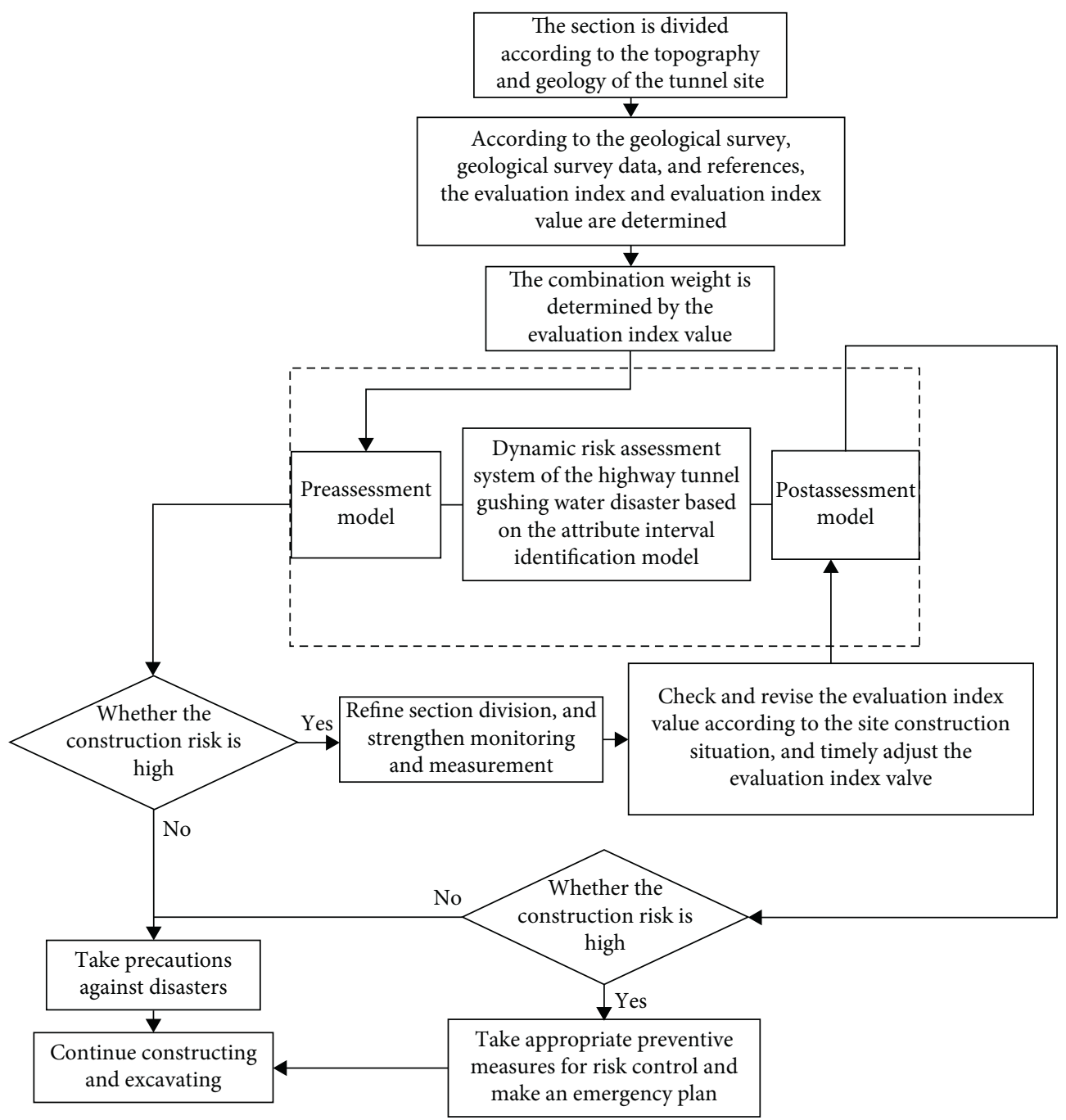

FIGURE 2: The highway tunnel gushing water dynamic risk assessment.

TABLE 5: Accident probability grading standards.

\begin{tabular}{lc}
\hline Risk level & Risk level description \\
\hline$R_{1}$ & Basically no risk \\
$R_{2}$ & Low risk \\
$R_{3}$ & Moderate risk \\
$R_{4}$ & High risk \\
\hline
\end{tabular}

\subsection{Risk before Assessment}

4.2.1. Determine the Evaluation Index and Its Value. Based on the geological exploration, the geological survey data, the advanced geological forecast, and the relevant reference, the evaluation index values of the target section $X_{i}(i=1,2,3,4,5)$ can be determined, which are combined with the grading standard of the highway tunnel water inrush risk assessment index in Table 2. It is shown in Table 7.

4.2.2. Weight Calculation. The combined weighting method is used to assign weights to each evaluation index. In the

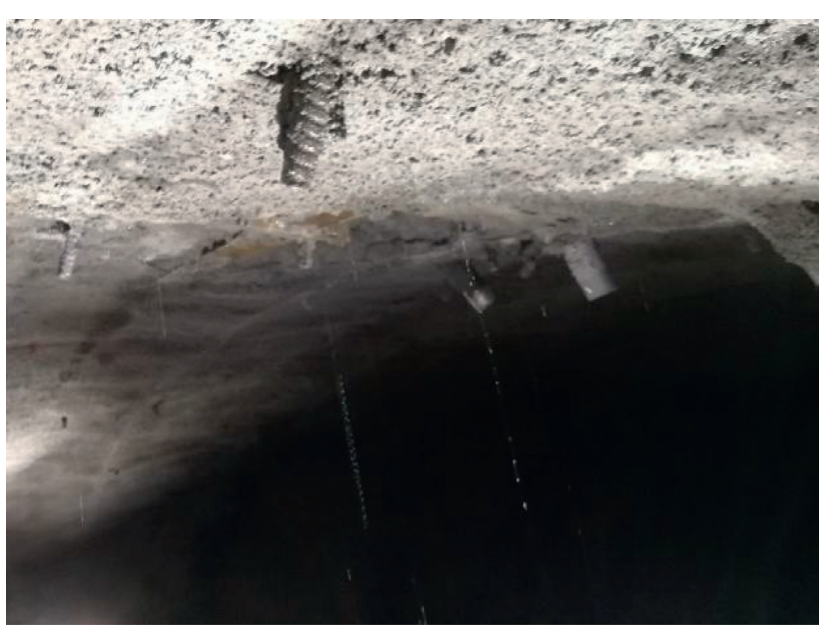

Figure 3: The tunnel water inrush of the arch roof after the initial support.

middle-long highway tunnel, the difference of the same evaluation index in different sections is large due to the topographic and geological conditions and other factors, 
TABle 6: Tunnel sections.

\begin{tabular}{lcc}
\hline Target segment & Sectional mileage range & Length (m) \\
\hline$X_{1}$ & $K 242+555 \sim K 242+635$ & 80 \\
$X_{2}$ & $K 242+635 \sim K 242+815$ & 180 \\
$X_{3}$ & $K 242+815 \sim K 242+875$ & 60 \\
$X_{4}$ & $K 242+875 \sim K 243+150$ & 275 \\
$X_{5}$ & $K 243+150 \sim K 243+337$ & 187 \\
\hline
\end{tabular}

TABle 7: Pre-evaluation evaluation index value.

\begin{tabular}{lcccccccccc}
\hline Target segment & $I_{1}$ & $I_{2}$ & $I_{3}$ & $I_{4}$ & $I_{5}$ & $I_{6}$ & $I_{7}$ & $I_{8}$ & $I_{9}$ & $I_{10}$ \\
\hline$X_{1}$ & 83 & 16 & 85 & 1.62 & {$[40,50]$} & {$[35,42]$} & {$[10,20]$} & 83 & 80 & {$[1,52.13]$} \\
$X_{2}$ & 72 & 25 & 180 & 1.62 & {$[300,330]$} & {$[26,38]$} & {$[30,40]$} & 85 & 82 & {$[77.625,340.05]$} \\
$X_{3}$ & 85 & 23 & 60 & 1.16 & {$[150,180]$} & {$[41,53]$} & {$[30,45]$} & 81 & 85 & {$[38.245,74.16]$} \\
$X_{4}$ & 78 & 35 & 275 & 1.62 & {$[1000,1300]$} & {$[55,62]$} & {$[45,55]$} & 90 & 92 & {$[57.37,131]$} \\
$X_{5}$ & 84 & 21 & 188 & 1.27 & {$[170,200]$} & {$[32,41]$} & {$[15,25]$} & 82 & 81 & {$[1,131]$} \\
\hline
\end{tabular}

which is a danger signal for the tunnel gushing water disaster. The entropy weight method is one way to determine the index weight according to the difference between the data. Therefore, the objective weight calculation uses the entropy weight method, which takes the pre-evaluation

$$
\alpha=\left\{\begin{array}{llllllllll}
0.002 & 0.034 & 0.130 & 0.010 & 0.489 & 0.024 & 0.085 & 0.001 & 0.001 & 0.224
\end{array}\right\}
$$

The subjective weight is calculated by the analytic hierarchy process, and the subjective weight of each evaluation index can be calculated as follows:

$$
\beta=\left\{\begin{array}{llllllllll}
0.061 & 0.132 & 0.121 & 0.235 & 0.091 & 0.048 & 0.111 & 0.133 & 0.034 & 0.035
\end{array}\right\}
$$

According to the objective weight and subjective weight calculated above and equation (24), the weight of each evaluation index can be obtained as follows:

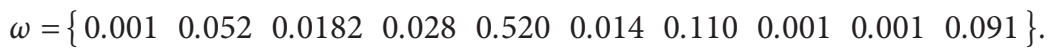

4.2.3. Preassessment. Combined with the single-index attribute measure function in Table 4 , the pre-evaluation evaluation index value in Table 7 , and combination weight $\omega$, the comprehensive attribute measure $\mu_{i k}$ of multiple indexes can be obtained by calculating from equations (3) to (16). The calculation results are shown in Table 8 . And it can be known from equation (25) as follows:

$$
k=\min \left\{l: \sum_{k=1}^{l} \mu_{i k} \geq 0 . \ddot{Y}_{5}, \quad 1 \leq l \leq 5\right\} .
$$

For the tunnel section $X_{1}$, the pre-evaluation results are shown as follows:

$$
\begin{array}{r}
\sum_{k=1}^{1} \mu_{1 k}=0.6120<0.65, \\
\sum_{k=1}^{2} \mu_{1 k}=0.6120+0.3405=0.9525>0.65 \\
\sum_{k=1}^{3} \mu_{1 k}=0.6120+0.3405+0.0465=0.999>0.65 \\
\sum_{k=1}^{4} \mu_{1 k}=0.6120+0.3405+0.0465+0.0010=1>0.65 .
\end{array}
$$


TABLE 8: Calculation table of attribute measure values.

\begin{tabular}{|c|c|c|c|c|c|}
\hline \multirow{2}{*}{ Target segment } & \multicolumn{4}{|c|}{ Attribute measure values } & \multirow{2}{*}{ Risk leve } \\
\hline & $R_{1}$ & $R_{2}$ & $R_{3}$ & $R_{4}$ & \\
\hline$X_{1}$ & 0.6120 & 0.3405 & 0.0465 & 0.0010 & $R_{2}$ \\
\hline$X_{2}$ & 0.0005 & 0.5869 & 0.2903 & 0.1224 & $R_{3}$ \\
\hline$X_{3}$ & 0.0983 & 0.7707 & 0.1114 & 0.0196 & $R_{3}$ \\
\hline$X_{4}$ & 0.0038 & 0.0895 & 0.2224 & 0.6842 & $R_{4}$ \\
\hline$X_{5}$ & 0.0423 & 0.7264 & 0.1610 & 0.0703 & $R_{2}$ \\
\hline
\end{tabular}

TABle 9: Tunnel sections.

\begin{tabular}{lr}
\hline Tunnel section $X_{4}$ & Sectional mileage range \\
\hline$X_{41}$ & $K 242+890 \sim K 242+900$ \\
$X_{42}$ & $K 243+010 \sim K 243+020$ \\
$X_{43}$ & $K 243+130 \sim K 243+140$ \\
\hline
\end{tabular}

TABle 10: Postevaluation evaluation index value.

\begin{tabular}{|c|c|c|c|c|c|c|c|c|c|c|}
\hline Tunnel section $X_{4}$ & $I_{1}$ & $I_{2}$ & $I_{3}$ & $I_{4}$ & $I_{5}$ & $I_{6}$ & $I_{7}$ & $I_{8}$ & $I_{9}$ & $I_{10}$ \\
\hline$X_{41}$ & 70 & 2.085 & 10 & 1.62 & {$[700,800]$} & {$[35,42]$} & {$[40,42]$} & 83 & 80 & {$[62.805,65.655]$} \\
\hline$X_{42}$ & 72 & 5.205 & 10 & 1.62 & {$[500,600]$} & {$[30,38]$} & {$[30,40]$} & 85 & 82 & {$[96.105,101.965]$} \\
\hline$X_{43}$ & 80 & 3.625 & 10 & 1.62 & {$[1000,1300]$} & {$[55,62]$} & {$[45,55]$} & 92 & 91 & {$[78.485,83.18]$} \\
\hline
\end{tabular}

Obviously, when $k$ is 2,3 , or 4 , all inequalities are true, so $k$ is 2 . The hazard risk level of the water inrush of the tunnel section $X_{1}$ is $R_{2}$. Similarly, the water gushing risks of tunnel intervals $X_{2}, X_{3}, X_{4}$, and $X_{5}$ are, respectively, $R_{3}, R_{3}, R_{4}$, and $R_{2}$. The final evaluation results are shown in Table 8 .

\subsection{Risk after Assessment}

4.3.1. Determine the Evaluation Index and Its Value. According to the pre-evaluation model, the highway tunnel water inrush risk in the tunnel section $X_{1}, X_{2}, X_{3}, X_{4}$, and $X_{5}$ is, respectively, $R_{2}, R_{3}, R_{3}, R_{4}$, and $R_{2}$. Tunnel sections $X_{2}$ and $X_{3}$ are of moderate risk in terms of water inrush, and tunnel section $X_{4}$ is of high risk in terms of water inrush. Therefore, tunnel sections $X_{2}, X_{3}$, and $X_{4}$ need to be segmented into smaller sections.

According to the on-site construction, the evaluation index value was modified in real time, and dynamically, the tunnel gushing water risk was evaluated in a more detailed way. Tunnel interval $X_{4}$ was taken as an example. So, it takes every $10 \mathrm{~m}$ as the section, and 3 sections of the tunnel are selected as the research object of postevaluation, as shown in Table 9.

Based on the topographic and geological conditions and the grading standards in Table 3, the evaluation index value of the target section of the tunnel can be determined as shown in Table 10.

4.3.2. Postassessment. Considering the single-index attribute measure function in Table 4, the postevaluation evaluation index value in Table 10, and the combination weight, the comprehensive attribute measure of multiple indexes can be obtained by calculating from equations (3) to (16). The calculation results are shown in Table 11. And it can be known from equation (25) as follows:

$$
k=\min \left\{l: \sum_{k=l}^{l} \mu_{i k} \geq 0.65, \quad 1 \leq l \leq 5\right\} .
$$

For tunnel section $X_{41}$, the postevaluation results are shown:

$$
\begin{array}{r}
\sum_{k=1}^{1} \mu_{4 k}=0.2538<0.65, \\
\sum_{k=1}^{2} \mu_{4 k}=0.2538+0.1075=0.3613<0.65 \\
\sum_{k=1}^{3} \mu_{4 k}=0.2538+0.1075+05921=0.9534>0.65, \\
\sum_{k=}^{4} \mu_{4 k}=0.2538+0.1075+0.5921+0.0466=1>0.65
\end{array}
$$

Obviously, when $k$ is equal to 3 or 4 , all inequalities are true, so $k$ is 3 . The hazard risk level of the water inrush of tunnel section $X_{41}$ is $R_{3}$. Similarly, the water gushing risks of tunnel intervals $X_{42}$ and $X_{43}$ are, respectively, $R_{3}$ and $R_{4}$. The water inrush hazard of tunnel intervals $K 242+890 \sim K 242+900$ and $K 243+010 \sim K 243+020$ is of moderate risk, while the water inrush hazard of tunnel interval $K 243+130 \sim K 243+140$ is of high risk. The final assessment results are shown in Table 11. 
TABLE 11: Calculation table of attribute measure values.

\begin{tabular}{lccccc}
\hline \multirow{2}{*}{ Tunnel section $X_{4}$} & \multicolumn{2}{c}{ Attribute measure values } & $R_{4}$ & Risk level \\
& $R_{1}$ & $R_{2}$ & $R_{3}$ & 0.0466 & $R_{3}$ \\
$X_{41}$ & 0.2538 & 0.1075 & 0.5921 & 0.0075 & $R_{3}$ \\
$X_{42}$ & 0.2332 & 0.2845 & 0.4748 & 0.5022 \\
$X_{43}$ & 0.2342 & 0.1080 & 0.1556 & $R_{4}$ \\
\hline
\end{tabular}
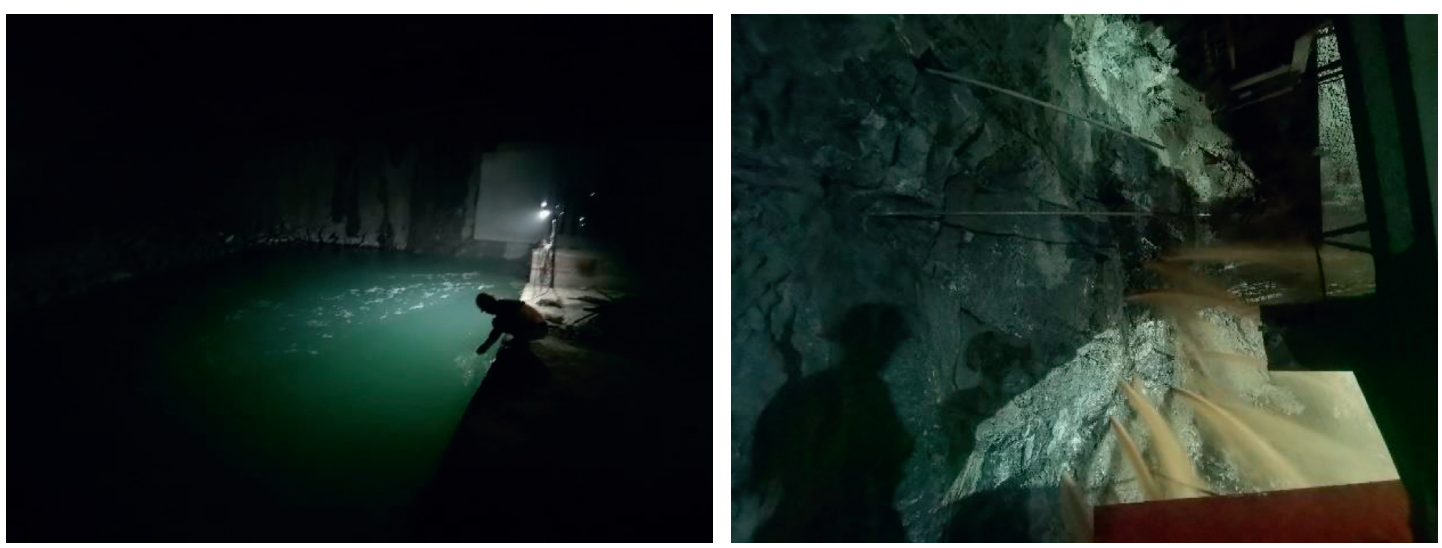

Figure 4: Verification of excavation results. (a) The lower steps were partially submerged by a surge of water. (b) Multiple jets of water spurted from the tunnel face.

TABle 12: Comparison of risk assessment results before and after excavation.

\begin{tabular}{lccccc}
\hline \multirow{2}{*}{ Tunnel section $X_{4}$} & \multicolumn{2}{c}{ Attribute measure values } & $R_{4}$ & Risk level \\
& $R_{1}$ & $R_{2}$ & $R_{3}$ & 0.5022 & $R_{4}$ \\
$X_{43}$ & 0.2342 & 0.1080 & 0.1556 & 0.5192 \\
$X_{43}^{\prime}$ & 0.2342 & 0.1080 & 0.1385 & $R_{4}$ \\
\hline
\end{tabular}

\section{Verification of Evaluation Results}

At about 7:20 a.m. on July 28, 2020, when the right line of the tunnel is excavated up to $K 243+132$, the water inrush occurred. Gushing water appeared in the gun holes at several positions of the tunnel surface with a certain pressure, and the jetting distance was about 3-5 $\mathrm{m}$. The water column on the right side of the tunnel surface was large, and some of it carried yellow mud, as shown in Figure 4.

After the accident occurred, the evaluation index values were modified according to the site construction conditions. The tunnel water inrush risk assessment was carried out again for the same section. Thus, it was concluded that tunnel section $K 243+130 \sim K 243+140$ was still of high risk. The evaluation results are in good agreement with the site construction. The comparison of risk assessment results before and after excavation is shown in Table 12 .

In this risk accident, the construction company has formulated emergency risk control measures for the gushing water disaster in the tunnel in advance and convened expert meetings in time to discuss the corresponding measures after the occurrence of danger. Therefore, no casualties were caused in this risk accident, and the construction loss was reduced to the minimum.

\section{Conclusion}

In this paper, a dynamic risk assessment based on the EWAHP attribute evaluation model is carried out for the highway tunnel water inrush under interval conditions.

(1) The index system and the index grading standards of the highway tunnel water inrush risk assessment have been established based on a highway tunnel case of the geological prospecting, geological survey data, and reference. Furthermore, a set of dynamic highway tunnel water inrush risk assessment models with preassessment model and postassessment model is established, which has combined with the combination of empowerment and the attribute identification theoretical model.

(2) The highway tunnel water gushing risk assessment model is set as a dynamic risk assessment model, which is based on the EW-AHP attribute evaluation model and composed of the pre-evaluation model and postevaluation model. The preassessment model is to control the water inrush risk of each tunnel section as a whole. Thus, the postassessment model is to carry out the risk assessment of the water inrush 
risk of the tunnel in time and dynamically according to the construction needs.

(3) The evaluation index values of the dynamic risk assessment model can be a certain value or range. So, it makes the evaluation index values more flexible, less restricted, and more in line with the engineering practice. Moreover, the weight calculation of each evaluation index only needs to be determined in the pre-evaluation model, and there is no need to change frequently in the postevaluation model due to the change of the evaluation index value.

(4) A case study of tunneling gushing water risk assessment was performed with the dynamic risk assessment model based on the attribute interval identification model. The water gushing risk of tunnel section $K 243+130 \sim K 243+140$ is $R_{4}$, which is of high risk. The evaluation results are consistent with the situation on the construction site, which verifies the rationality and feasibility of the evaluation model.

\section{Data Availability}

The datasets used and analyzed during the current study are available from the corresponding author upon reasonable request.

\section{Conflicts of Interest}

The authors declare no conflicts of interest.

\section{Acknowledgments}

The authors would like to express appreciation and thanks to the managers and Sanming Puyan Expressway Co., Ltd. This research was funded by the Natural Science Foundation of China (51678164 and 51478118), the Guangxi Natural Science Foundation Program (2018GXNSFDA138009), the Guangxi Science and Technology Plan Projects (AD18126011), the Scientific Research Foundation of Guangxi University (XTZ160590), and the Project supported by GDHVPS(2019).

\section{References}

[1] M. S. Wang, "Development overview of railway tunnels and underground space in China," Tunnel Construction, vol. 30, no. 4 , pp. 351-364, 2010, in Chinese.

[2] S.-S. Lin, S.-L. Shen, A. Zhou, and Y.-S. Xu, "Novel model for risk identification during karst excavation," Reliability Engineering \& System Safety, vol. 209, p. 107435, 2021.

[3] S.-S. Lin, S.-L. Shen, A. Zhou, and Y.-S. Xu, "Risk assessment and management of excavation system based on fuzzy set theory and machine learning methods," Automation in Construction, vol. 122, p. 103490, 2021.

[4] L. Li, X. B. Chen, J. X. Cheng et al., "Study on model test of rockburst prevention for water inrush in deep and long tunnels," Chinese Journal of Rock Mechanics and Engineering, vol. 39, no. S2, pp. 3278-3285, 2020, in Chinese.
[5] Z. Zhu, X. C. Wang, Y. C. Yuan, and X. L. Wang, "Risk assessment of karst tunnel inrush water based on weighted average method," Highway Engineering, vol. 40, no. 6, pp. 51-54, 2015, in Chinese.

[6] Q. Li and L. Ning, "Research and application of theoretical model of attribute interval identification," Mathematics in Practice and Theory, vol. 1, pp. 50-54, 2002, in Chinese.

[7] Z. Q. . Zhou, S. C. Li, L. P. Li, S. S. Shi, S. G. Song, and $\mathrm{K}$. Wang, "Attribute identification model and its engineering application for karst tunnel risk assessment of inrush water," Rock and Soil Mechanics, vol. 34, no. 3, pp. 818-826, 2013, in Chinese.

[8] C. P. Wen, "attribute identification model and example for gas outburst risk assessment in tunnels," Journal of China Coal Society, vol. 36, no. 8, pp. 1322-1328, 2011, in Chinese.

[9] S. Wang, L.-p. Li, S. Cheng, H. Hu, H. Jin, and S. Gao, "Dynamic risk assessment method of tunnel collapse based on attribute interval assessment model and application," Polish Journal of Environmental Studies, vol. 29, no. 5, pp. 38533864, 2020.

[10] Q. Zhang, K. Liu, and Z. Y. Gao, "Application of attribute comprehensive evaluation system in urban traffic planning," Systems Engineering-Theory \& Practice, vol. 22, no. 6, pp. 113-120, 2002, in Chinese.

[11] Q. T. Pei, H. B. Li, and Y. Q. Liu, "Evaluation model and application of rock burst tendency prediction ash based on combination weight," Rock and Soil Mechanics, vol. 35, no. S1, pp. 49-56, 2014, in Chinese.

[12] H. Liu, Y. Dong, and F. Wang, "Gas outburst prediction model using improved entropy weight grey correlation analysis and IPSO-LSSVM," Mathematical Problems in Engineering, vol. 2020, Article ID 8863425, 10 pages, 2020.

[13] J. Zhang, "A study on mental health assessments of college students based on triangular fuzzy function and entropy weight method," Mathematical Problems in Engineering, vol. 2021, Article ID 6659990, 8 pages, 2021.

[14] W. Gong, N. Wang, N. Zhang, W. Han, and H. Qiao, "Water resistance and a comprehensive evaluation model of magnesium oxychloride cement concrete based on Taguchi and entropy weight method," Construction and Building Materials, vol. 260, p. 119817, 2020.

[15] F. Li, K. K. Phoon, X. Du, and M. Zhang, "Improved AHP method and its application in risk identification," Journal of Construction Engineering and Management, vol. 139, no. 3, pp. 312-320, 2013.

[16] T. L. Saaty, "A scaling method for priorities in hierarchical structures," Journal of Mathematical Psychology, vol. 15, no. 3, pp. 234-281, 1977.

[17] G.-F. Wang, H.-M. Lyu, J. Shen, L.-H. Lu, G. Li, and A. Arulrajah, "Evaluation of environmental risk due to metro system construction in jinan, China," International Journal of Environmental Research and Public Health, vol. 14, no. 10, p. 1114, 2017.

[18] W. Chen, X. J. Liu, and Y. J. Xia, "Multi-factor credit evaluation model of internet of vehicles based on the analytic hierarchy process," Journal of Zhejiang University (Engineering Science), vol. 54, no. 4, pp. 722-731, 2020, in Chinese.

[19] Z. X. Qiu, J. Q. Ju, D. Ren, D. Y. Cui, and C. J. Liu, "Optimization design of machine tool beam based on orthogonal test and combination-weighted grey correlation," Journal of Vibration and Shock, vol. 36, no. 12, pp. 105-111, 2017, in Chinese.

[20] Z. Q. Zhou, J. Kong, W. M. Yang et al., "Improved attribute interval Identification method and its application in the risk 
assessment of tunnel water inrush," Journal of Central South University (Science and Technology), vol. 51, no. 6, pp. 17031711, 2020, in Chinese.

[21] S. C. Li, Z. Q. Zhou, L. P. Li, S. S. Shi, and Z. H. Xu, “Theory and Method of water inrush risk assessment of Karst tunnel and its Engineering application," Journal of Central South University (Science and Technology), vol. 32, no. 9, pp. 1858-1867, 2013, in Chinese.

[22] B. Y. Mao, M. Xu, and L. W. Jiang, "Preliminary study on the risk assessment of tunnel karst water inrush and mud inrush," Carsologica Sinica, vol. 29, no. 2, pp. 183-189, 2010, in Chinese.

[23] Ministry of Housing and Urban-Rural Development of the People's Republic of China, Code for Geological Investigation of Water Conservancy and Hydropower Projects (GB504872008), China Planning Press, Beijing, China, 2009.

[24] Department of Transportation Engineering Quality Supervision Bureau, Highway Bridge and Tunnel Engineering Construction Risk Safety Assessment System and Guidelines Analysis, China Communications Press, Beijing, China, 2011, in Chinese. 